\title{
Detection of AR-V7 mRNA in whole blood may not predict the effectiveness of novel endocrine drugs for castration-resistant prostate cancer
}

This article was published in the following Dove Press journal:

Research and Reports in Urology

28 January 2016

Number of times this article has been viewed

\section{Takumi Takeuchi \\ Yumiko Okuno \\ Mami Hattori-Kato \\ Masayoshi Zaitsu \\ Koji Mikami}

Department of Urology, Kanto Rosai Hospital, Kawasaki, Japan
Correspondence: Takumi Takeuchi Department of Urology, Kanto Rosai Hospital, I-I Kizukisumiyoshi-cho, Nakahara-ku, Kawasaki 2I I-85 I0, Japan Tel +8 I 444 | | 3|3 |

Fax +8I 444333150

Email takeuchit@abelia.ocn.ne.jp

\begin{abstract}
A splice variant of androgen receptor (AR), AR-V7, lacks in androgen-binding portion and leads to aggressive cancer characteristics. Reverse transcription-polymerase chain reactions (PCRs) and subsequent nested PCRs for the amplification of AR-V7 and prostate-specific antigen (PSA) transcripts were done for whole blood of patients with prostate cancer and male controls. With primary reverse transcription PCRs, AR-V7 and PSA were detected in $4.5 \%$ and $4.7 \%$ of prostate cancer, respectively. With nested PCRs, AR-V7 messenger RNA (mRNA) was positive in $43.8 \%$ of castration-sensitive prostate cancer and $48.1 \%$ of castration-resistant prostate cancer (CRPC), while PSA mRNA was positive in $6.3 \%$ of castration-sensitive prostate cancer and $18.5 \%$ of CRPC. Whole-blood samples of controls showed AR-V7 mRNA expression by nested PCR. Based on multivariate analysis, expression of AR-V7 mRNA in whole blood was not significantly correlated with clinical parameters and PSA mRNA in blood, while univariate analysis showed a correlation between AR-V7 mRNA and metastasis at initial diagnosis. Detection of AR-V7 mRNA did not predict the reduction of serum PSA in patients with CRPC following abiraterone and enzalutamide administration. In conclusion, AR-V7 mRNA expression in normal hematopoietic cells may have annihilated the manifestation of aggressiveness of prostate cancer and the prediction of the effectiveness of abiraterone and enzalutamide by the assessment of AR-V7 mRNA in blood.
\end{abstract}

Keywords: AR-V7, prostate cancer, castration-resistant

\section{Introduction}

Androgen deprivation therapy for prostate cancer first reported by Huggins ${ }^{1}$ is effective at first, but eventually castration-resistant prostate cancer (CRPC) occurred and often caused cancer death. The annual incidence of and death from prostate cancer in Japan are 126.6 and 18.9 per 1,000,000 men, respectively, and both are still growing. ${ }^{2,3}$ Novel endocrine drugs for CRPC, abiraterone acetate and enzalutamide, have been introduced and shown to prolong overall survival of patients with CRPC regardless of preceding docetaxel therapy. ${ }^{4-7}$ Abiraterone acetate is a potent and irreversible inhibitor of CYP17 (17 $\alpha$-hydroxylase/C17, 20-lyase) that suppresses androgen synthesis, while enzalutamide is an androgen receptor (AR) signaling inhibitor in addition to being a highly potent AR antagonist.

Recently, splice variants of AR were identified. One of them, AR-V7, lacks the androgen-binding portion but automatically transduces intracellular signals resulting in enhanced nuclear transcription and more aggressive characteristics of cancer. ${ }^{8,9}$ Detection of AR-V7 messenger RNA (mRNA) in circulating tumor cells (CTCs) in patients with CRPC is reported to predict the noneffectiveness of 
abiraterone acetate and enzalutamide. In the report, it is described that AR-V7 is specific to tumor cells. ${ }^{10}$ If AR-V7 is tumor specific, it is not absolutely necessary to isolate CTCs from whole blood, and thus mRNA extracted from whole blood may be sufficient to detect AR-V7 mRNA in CTCs.

Here, we assessed the AR-V7 mRNA expression in whole blood of patients with prostate cancer and analyzed its linkage with clinical parameters and effectiveness of abiraterone acetate and enzalutamide.

\section{Materials and methods}

Whole blood of 43 patients (age 62-94 years, median 78 years) with prostate cancer and six male controls (age 32-79 years, median 53 years) without prostate cancer was collected in tubes containing ethylenediaminetetraacetic acid. Thirty-five of them had been on androgen deprivation therapy, while others were scheduled to undergo the therapy. Abiraterone acetate or enzalutamide was administered to eleven patients with CRPC. For those patients, AR-V7 in blood was measured before abiraterone acetate or enzalutamide administration. Total RNA was extracted from $0.4 \mathrm{~mL}$ of whole blood using oligo dT primer and a DNA Extractor ${ }^{\circledR}$ WB-Rapid Kit (Wako Pure Chemical Industries, Ltd, Osaka, Japan). Complementary DNA was synthesized from total RNA using a PrimeScript ${ }^{\mathrm{TM}}$ II first strand cDNA Synthesis Kit (Takara Bio, Inc., Kusatsu, Japan). Thirty-five cycles of polymerase chain reactions (PCRs) (primary PCR) in $50 \mu \mathrm{L}$ were performed using a Premix Taq DNA Polymerase (Takara Bio, Inc.) for the amplification of AR-V7, prototype AR, prostate-specific antigen (PSA), and glyceraldehyde 3-phosphate dehydrogenase transcripts. In addition, 30 cycles of nested PCRs for AR-V7, prototype AR, and PSA were conducted using $1 \mu \mathrm{L}$ of primary PCR products by substituting each corresponding antisense primer. The PCR primers are listed in Table 1. PCR amplicons were electrophoresed in a $1.5 \%$ agarose gel.

For univariate and multivariate analyses, odds ratios of positive expression of AR-V7 mRNA in whole blood were estimated, adjusting with metastasis at diagnosis, Gleason score, initial PSA, duration of hormone therapy, presence of CRPC, presence of clinical recurrence, and detection of PSA mRNA in whole blood by using logistic regression models. A waterfall plot was drawn to show the maximum reduction rate of PSA after abiraterone acetate and enzalutamide administration to metastatic patients with CRPC.

The experiment was conducted with the human subjects' understanding and written informed consent was obtained. The Ethical Committee of Kanto Rosai Hospital approved this study and the experiments.

\section{Results \\ Patients with prostate cancer}

Clinical characteristics of patients with prostate cancer are listed in Table 2. With primary PCRs, glyceraldehyde 3-phosphate dehydrogenase mRNA was positive in all wholeblood samples of patients with prostate cancer, while prototype AR, AR-V7, and PSA were detected in 53.3\%, 4.5\%, and $4.7 \%$ of whole-blood samples, respectively. With nested PCR, AR-V7 mRNA was positive in $43.8 \%$ of patients with castration-sensitive prostate cancer and $48.1 \%$ of patients with CRPC, while PSA mRNA was positive in $6.3 \%$ of castrationsensitive prostate cancer and $18.5 \%$ of CRPC. Prototype AR mRNA was detected in $93.8 \%$ of samples. Representative data are shown in Figure 1. With multivariate analysis, expression of AR-V7 mRNA in whole blood was not significantly correlated with metastasis at diagnosis, Gleason score, initial PSA, duration of hormone therapy, presence of CRPC, presence of clinical recurrence, or detection of PSA mRNA in whole blood as shown in Table 3. Univariate analysis showed that AR-V7

Table I Primers for PCR amplification

\begin{tabular}{llll}
\hline Product & Type of primer & Sequence (5' to $\mathbf{3}^{\prime}$ ) & Reference \\
\hline GAPDH & Sense & GCCATCAATGACCCCTTCATT & 12 \\
GAPDH & Antisense & TTGACGGTGCCATGGAATTT & 12 \\
Proto AR & Sense & CCATCTTGTCGTCTTCGGAAATGTTATGAAGC & 8 \\
Proto AR & Antisense & AGCTTCTGGGTTGTCTCCTCAGTGG & 8 \\
Proto AR & Antisense for nested PCR & TCTGGGTTGTCTCCTCAGTGGGGCT & NA \\
AR-V7 & Sense & CCATCTTGTCGTCTTCGGAAATGTTATGAAGC & 8 \\
AR-V7 & Antisense & TTTGAATGAGGCAAGTCAGCCTTTCT & 8 \\
AR-V7 & Antisense for nested PCR & GAATGAGGCAAGTCAGCCTTTCTTCA & NA \\
PSA & Sense & CAGTCTGCGGCGGTGTT & I3 \\
PSA & Antisense & GCAAGATCACGCTTTTGTTCCT & I3 \\
PSA & Antisense for nested PCR & AGATCACGCTTTTGTTCCTGAT & NA \\
\hline
\end{tabular}

Abbreviations: GAPDH, glyceraldehyde 3-phosphate dehydrogenase; Proto, prototype; AR, androgen receptor; PSA, prostate-specific antigen; PCR, polymerase chain reaction; NA, not applicable. 
Table 2 Characteristics of prostate cancer patients

\begin{tabular}{ll}
\hline Characteristics & \\
\hline Age at diagnosis (years), range (median) & $59-88$ (73) \\
Metastasis at diagnosis & 17 \\
$\quad$ Negative & 26 \\
$\quad$ Positive & \\
Biopsy Gleason score & 4 \\
$\quad$ GS $\leq 6$ & 5 \\
GS 7 & 31 \\
GS 8-10 & $5.3-9,529$ (I30) \\
iPSA (ng/mL), range (median) & \\
Sensitivity to castration & 20 \\
$\quad$ CSPC & 23 \\
CRPC & $0.0-14.1$ (2.5) \\
Duration of hormonal T (years), & \\
range (median) & \\
Clinical recurrence & 35 \\
$\quad$ Negative & 8 \\
Positive & \\
Previous docetaxel T & 37 \\
$\quad$ Negative & 6 \\
Positive
\end{tabular}

Notes: Gleason score is missing for three patients, as pathologist did not report it. Abbreviations: iPSA, initial prostate-specific antigen; CSPC, castration-sensitive prostate cancer; CRPC, castration-resistant prostate cancer; T, therapy.

mRNA was correlated with metastasis at initial diagnosis, but not with other parameters (Table 3).

A waterfall plot analysis did not confirm whether detection of AR-V7 mRNA in whole blood could predict the reduction of serum PSA in patients with CRPC following abiraterone acetate or enzalutamide administration (Figure 2). Nondetection of AR-V7 mRNA was not correlated with the reduction of serum PSA ( $P=0.8865$ by the two-tailed chi-square test).

\section{Controls without prostate cancer}

Whole-blood samples of controls without prostate cancer similarly processed showed AR-V7 mRNA expression by nested PCR as shown in Figure 3.

\section{Discussion}

In the present study, the detection rate of AR-V7 mRNA in blood was much higher than that of PSA, although not all CTCs in patients with prostate cancer may express PSA mRNA. Additionally, expression of AR-V7 mRNA in blood did not correlate with that of PSA mRNA in blood. Therefore, there is a high possibility that AR-V7 mRNA is expressed not only in CTCs but also in nontumorous cells in blood, that is, hematopoietic cells. Prototype AR mRNA is known to be expressed in various types of cells including hematopoietic cells, so it is quite reasonable to suppose that splice variants of AR mRNA are also expressed in hematopoietic cells. ${ }^{11}$ Actually, AR-V7 mRNA was detected in whole-blood samples of controls without prostate cancer.

It is supposed that prostate cancer cells expressing AR-V7 have more aggressive characteristics, but AR-V7 mRNA

Table 3 Factors contributing to positive AR-V7 mRNA in blood

\begin{tabular}{|c|c|c|c|c|c|c|}
\hline \multirow[t]{2}{*}{ Clinical parameters } & \multicolumn{2}{|c|}{ Positive AR-V7 mRNA } & \multicolumn{2}{|c|}{ Multivariate } & \multicolumn{2}{|c|}{ Univariate } \\
\hline & $\mathbf{n}$ & $\%$ & OR & $P$-value & OR & $P$-value \\
\hline \multicolumn{7}{|l|}{ Metastasis at diagnosis } \\
\hline Negative & 17 & 23.5 & & & & \\
\hline Positive & 26 & 61.5 & 4.90 & 0.0770 & 5.20 & 0.0146 \\
\hline \multicolumn{7}{|l|}{ Gleason score } \\
\hline$\leq 7$ & 9 & 33.3 & & & & \\
\hline $8-10$ & 31 & 51.6 & 1.08 & 0.9339 & 2.13 & 0.3337 \\
\hline \multicolumn{7}{|l|}{ iPSA (ng/mL) } \\
\hline $0-20$ & 13 & 38.5 & & & & \\
\hline$\geq 20$ & 30 & 50.0 & 0.65 & 0.6437 & 1.60 & 0.4860 \\
\hline \multicolumn{7}{|c|}{ Sensitivity to castration } \\
\hline CSPC & 16 & 43.8 & & & & \\
\hline CRPC & 27 & 48.1 & 0.68 & 0.6292 & 1.19 & 0.7799 \\
\hline \multicolumn{7}{|c|}{ Duration of hormonal T (years) } \\
\hline $0-3$ & 23 & 52.2 & & & & \\
\hline$\geq 3$ & 19 & 42.1 & 1.28 & 0.7424 & 0.67 & 0.5155 \\
\hline \multicolumn{7}{|l|}{ Clinical recurrence } \\
\hline Negative & 35 & 45.7 & & & & \\
\hline Positive & 8 & 50.0 & 0.92 & 0.9332 & 1.19 & 0.8264 \\
\hline \multicolumn{7}{|l|}{ PSA mRNA in WB } \\
\hline Negative & 37 & 43.2 & & & & \\
\hline Positive & 6 & 66.7 & 4.16 & 0.2998 & 2.63 & 0.2860 \\
\hline
\end{tabular}

Abbreviations: AR, androgen receptor; mRNA, messenger RNA; iPSA, initial prostate-specific antigen; CSPC, castration-sensitive prostate cancer; CRPC, castration-resistant prostate cancer; T, therapy; WB, whole blood; multivariate, multivariate analysis; univariate, univariate analysis; OR, odds ratio. 


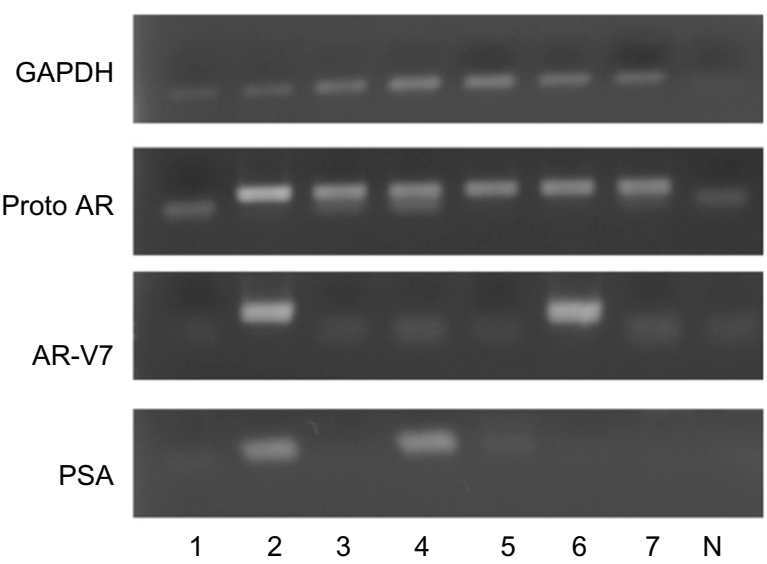

Figure I Detection of AR-V7 mRNA in whole blood of patients with CRPC (lanes I-7).

Notes: GAPDH: 35 cycles of primary PCR; Proto AR (prototype AR); AR-V7; PSA: 30 cycles of nested PCR following 35 cycles of primary PCR; N: negative control (no genomic DNA added).

Abbreviations: AR, androgen receptor; mRNA, messenger RNA; CRPC, castrationresistant prostate cancer; GAPDH, glyceraldehyde 3-phosphate dehydrogenase; $\mathrm{PCR}$, polymerase chain reaction; PSA, prostate-specific antigen.

expression in blood did not necessarily indicate aggressive parameters of prostate cancer such as high Gleason scores, high initial serum PSA levels, acquisition of castration resistance, or clinical recurrence in the present study. Only positive metastasis at diagnosis was linked with positive AR-V7 mRNA in blood. The AR-V7 mRNA expression in normal hematopoietic cells may have obscured the manifestation of aggressiveness of prostate cancer by the assessment of AR-V7 mRNA in blood.

In contrast to the preceding report, ${ }^{10}$ the resistance to further androgen-related cancer therapy in cases with positive AR-V7 mRNA in blood was not observed in the present study. AR-V7 mRNA expression in normal hematopoietic cells may have annihilated the prediction of

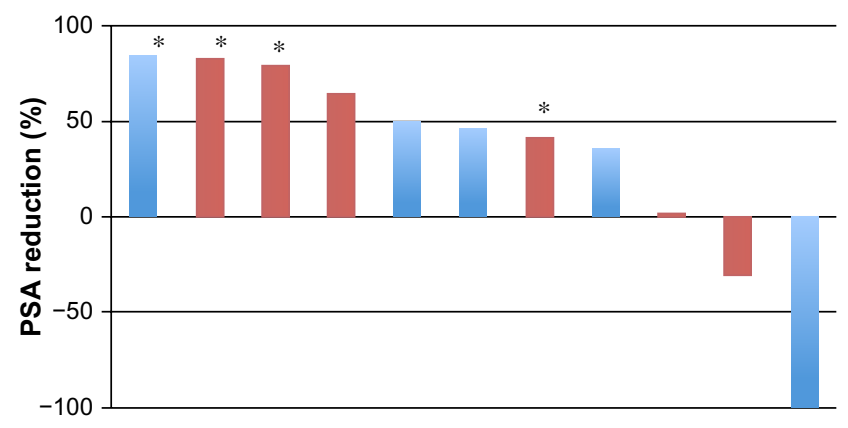

Figure 2 Waterfall plot showing maximum reduction rate of PSA following abiraterone acetate and enzalutamide administration to metastatic patients with CRPC.

Notes: Blue bars indicate negative AR-V7 mRNA in whole blood; red bars indicate positive AR-V7 mRNA; asterisks represent cases administered abiraterone acetate. Abbreviations: PSA, prostate-specific antigen; CRPC, castration-resistant prostate cancer;AR, androgen receptor; mRNA, messenger RNA.

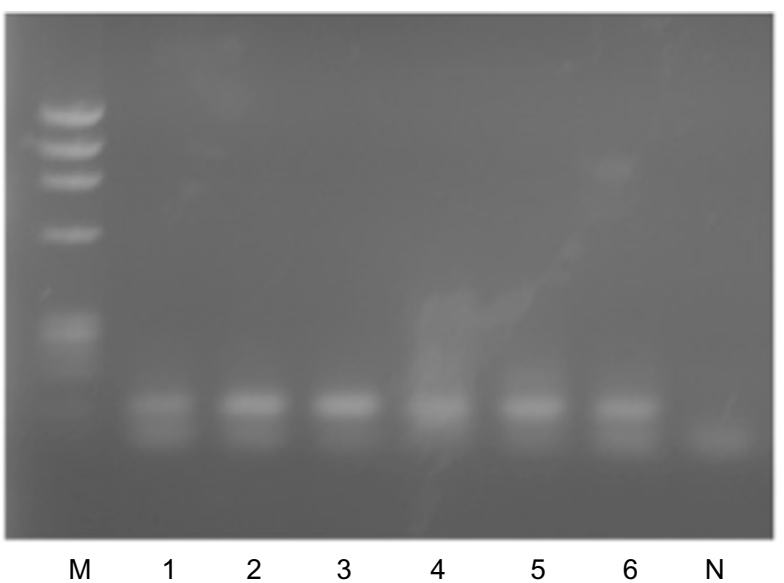

Figure 3 Detection of AR-V7 mRNA in whole blood of controls without prostate cancer (lanes I-6).

Notes: Thirty cycles of nested PCR following 35 cycles of primary PCR; M: $\varphi$ XI74/ Hae III Marker; N: negative control (no genomic DNA added).

Abbreviations: AR, androgen receptor; $m R N A$, messenger RNA; PCR, polymerase chain reaction.

the effectiveness of abiraterone acetate and enzalutamide for CRPC. ${ }^{10}$

AR-V7 expression was first described in metastatic tissue of hormone-resistant prostate cancer. ${ }^{8}$ If AR-V7 is expressed in normal hematopoietic cells, it may be mandatory to completely eliminate infiltrating hematopoietic cells from cancercontaining tissue before conducting AR-V7 assays in order to show that AR-V7 of tumor origin is actually expressed. This maneuver seems much more difficult in solid tissues than in liquid materials like blood. Moreover, the amounts of AR-V7 mRNA in cells in blood are very low, as a lot of cycles of amplification are often necessary to show up AR-V7 transcripts. The threshold for positive AR-V7 detection could be very hard to determine. Considering the factors mentioned earlier, it may not be very convenient to utilize AR-V7 mRNA expression as a biomarker for abiraterone acetate and enzalutamide administration in patients with CRPC.

\section{Conclusion}

AR-V7 mRNA expression in normal hematopoietic cells may annihilate the manifestation of aggressiveness of prostate cancer and the prediction of the effectiveness of abiraterone and enzalutamide for CRPC by assessment of AR-V7 mRNA in blood.

\section{Disclosure}

The authors report no conflicts of interest in this work, and no conflict of interest regarding the publication of this paper.

\section{References}

1. Huggins C. Effect of orchiectomy and irradiation on cancer of the prostate. Ann Surg. 1942;115(6):1192-1200. 
2. Matsuda A, Matsuda T, Shibata A, et al. Cancer incidence and incidence rates in Japan in 2008: a study of 25 population-based cancer registries for the monitoring of cancer incidence in Japan (MCIJ) project. Jpn J Clin Oncol. 2014;44(4):388-396.

3. Vital Statistics Japan. Ministry of Health, Labour and Welfare.

4. Fizazi K, Scher H, Molina A, et al. Abiraterone acetate for treatment of metastatic castration-resistant prostate cancer: final overall survival analysis of the COU-AA-301 randomised, double-blind, placebo-controlled phase 3 study. Lancet Oncol. 2012;13(10):983-992.

5. Ryan C, Smith MR, de Bono JS, et al. Abiraterone in metastatic prostate cancer without previous chemotherapy. $N$ Engl J Med. 2013;368(2):138-148

6. Scher HI, Fizazi K, Saad F, et al. Increased survival with enzalutamide in prostate cancer after chemotherapy. $N$ Engl J Med. 2012;367(13): 1187-1197.

7. Beer TM, Armstrong AJ, Rathkopf DE, et al. Enzalutamide in metastatic prostate cancer before chemotherapy. N Engl J Med. 2014; 371(5):424-433.

8. Hu R, Dunn TA, Wei S, et al. Ligand-independent androgen receptor variants derived from splicing of cryptic exons signify hormone-refractory prostate cancer. Cancer Res. 2009;69(1):16-22.
9. Hu R, Isaacs WB, Luo J. A snapshot of the expression signature of androgen receptor splicing variants and their distinctive transcriptional activities. Prostate. 2011;71(15):1656-1667.

10. Antonarakis ES, Lu C, Wang H, et al. AR-V7 and resistance to enzalutamide and abiraterone in prostate cancer. N Engl J Med. 2014;371(11): 1028-1038.

11. BioGPS. [webpage on the Internet]. Androgen Receptor. Available from: http://biogps.org/\#goto=genereport\&id=367. Accessed December 22, 2015 .

12. Akasaka E, Nakano H, Sato-jin K, et al. The interaction of photoprotective effects between keratinocytes and melanocytes via endothelin-1/ stem cell factor signaling. Hirosaki Med J. 2011;62(2-4):138-143.

13. Yates DR, Rouprêt M, Drouin SJ, et al. Quantitative RT-PCR analysis of PSA and prostate-specific membrane antigen mRNA to detect circulating tumor cells improves recurrence-free survival nomogram prediction after radical prostatectomy. Prostate. 2012;72(12):1382-1388.
Research and Reports in Urology

\section{Publish your work in this journal}

Research and Reports in Urology is an international, peer-reviewed, open access journal publishing original research, reports, editorials, reviews and commentaries on all aspects of adult and pediatric urology in the clinic and laboratory including the following topics: Pathology, pathophysiology of urological disease; Investigation and treatment of

\section{Dovepress}

urological disease; Pharmacology of drugs used for the treatment of urological disease. The manuscript management system is completely online and includes a very quick and fair peer-review system, which is all easy to use. Visit http://www.dovepress.com/testimonials.php to read real quotes from published authors.

Submit your manuscript here: http://www.dovepress.com/research-and-reports-in-urology-journal 